Special Issue of the 6th International Congress \& Exhibition (APMAS2016), Maslak, Istanbul, Turkey, June 1-3, 2016

\title{
Prediction of First Order Focusing Properties of Ideal Hemispherical Deflector Analyzer Using Artificial Neural Network
}

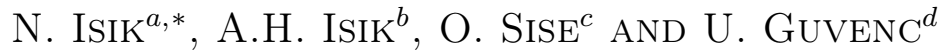 \\ ${ }^{a}$ Mehmet Akif Ersoy University, Department of Science Education, 15030 Burdur, Turkey \\ ${ }^{b}$ Mehmet Akif Ersoy University, Department of Computer Engineering, 15030 Burdur, Turkey \\ ${ }^{c}$ Süleyman Demirel University, Department of Science Education, 32260 Isparta, Turkey \\ ${ }^{d}$ Duzce University, Department of Electrical and Electronics Engineering, 81620 Duzce, Turkey
}

\begin{abstract}
Electrostatic energy analyzers are irreplaceable instruments to analyze the electron beams energies. In this context, the knowledge of electron trajectories in electrostatic energy analyzers has major importance in collision physics as well as in different scientific instruments for surface science. In this study, electron trajectories for different energies in an ideal field $180^{\circ}$ hemispherical deflector analyzer are investigated by artificial neural network prediction method. The SIMION 8.1 simulation program is used as a data source for training and testing of artificial neural network. Artificial neural network based prediction has been performed using Matlab R2012b program. Obtained performance results indicate that this approach provides new perspectives for the rapid solution to the problems in charged particle optics.
\end{abstract}

DOI: 10.12693/APhysPolA.131.10

PACS/topics: 42.79.Fm, 07.05.Tp

\section{Introduction}

In many branches of collision physics, it is required to analyze the energies of the collected electrons emanating from the interaction region. As a solution, hemispherical deflector analyzers (HDAs) are designed as vital devices to disperse electrons depending on their kinetic energies [1-5]. To date, the conventional methods have been performed to determine whether a particle is focused at the entrance and exit of the hemispheres [6,7]. Most of the earlier studies are focused on the ideal field analyzers for analytic determination of electron paths within the hemispheres [8]. However, the fringing fields at the entrance and the exit of the hemispheres lead to the angular aberrations in a conventional HDA. For the elimination of these effects, the fringing field correctors are performed [9-13]. In the absence of any fringing fields, the ideal HDAs indicate the first order focusing characteristics. In recent years, successful applications are performed for solving problems in charged particle optics using artificial neural networks (ANNs) in prediction $[14,15]$ and classification [16-18]. ANNs are parallel computing systems with interconnected processors. These networks have a learning ability inspired by human brain. With this ability, the network can solve complex problems from optimum training patterns. In this study, the focusing action of the ideal field HDA is determined at the entry and exit of the hemispheres by using ANN method for different $\gamma$ values. Training data set is obtained from

*corresponding author; e-mail: nimetok@hotmail.com
SIMION 8.1 program. The performance of the method is tested by the blinded data that the network has not seen before.

This study is organized as follows. Section 2 provides detailed information about ideal field HDA. Test results of the trained network with obtained data using SIMION 8.1 simulation program are presented in Sect. 3 .

\section{Ideal field hemispherical deflector analyzer}

The HDA used to analyze the energies of electrons consists of an input electrostatic lens system and two concentric hemispherical electrodes which are held at different potentials. These potential differences create a central $1 / r^{2}$ field within the hemispheres when the fringing fields are neglected. Using the path determined by the potentials, charged particles are separated according to their kinetic energies. A schematic representation of the HDA geometry is shown in Fig. 1a. Electron paths for different $\alpha$ values are also given in Fig. $1 \mathrm{~b}$.

An ideal HDA is described with no fringing fields and the force on the charged particle is proportional to ideal $1 / r^{2}$ field. Depending on the radius of inner $\left(R_{1}\right)$ and outer $\left(R_{2}\right)$ hemispheres, the potential is calculated by

$$
V_{(r, \theta, \phi)}=V_{r}=-\frac{\left(\frac{\Delta V}{\Delta R}\right) R_{1} R_{2}}{r}+\frac{V_{2} R_{2}-V_{1} R_{1}}{\Delta R},
$$

where $\Delta V=V_{2}-V_{1}$ and $\Delta R=R_{2}-R_{1}$.

The HDA focusing properties can be determined for a given initial and final radius at the entrance and exit of the hemispheres. In an ideal HDA, the charged particles with kinetic energy $T$ enter the lens region and are focused at the HDA entry $\left(R_{0}\right)$ and the central path of charged particle beam exits at $R_{\pi}$. The central trajectory for the conventional centric HDA is a circle 

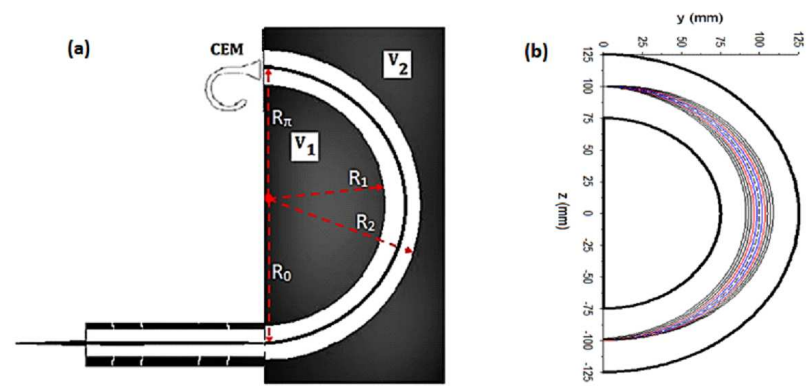

Fig. 1. (a) The schematic view of $180^{\circ}$ hemispherical deflector analyzer. CEM - channel electron multiplier detector. (b) The electron trajectories within the hemispheres [13].

$\left(R_{0}=R_{\pi}=\bar{R}\right) . \quad E$ is the pass energy of the HDA. In this context, the particle trajectory is calculated by

$$
\begin{aligned}
r_{(\theta)} & =r_{0}\left[\frac{\left(\frac{\Delta V}{\Delta R}\right) q R_{1} R_{2}(1-\cos \theta)}{2 r_{0} \cos _{\alpha}^{2}\left(E-q \frac{V_{2} R_{2}-V_{1} R_{1}}{\Delta R}\right)+\frac{q\left(\frac{\Delta V}{\Delta R}\right) R_{1} R_{2}}{r_{0}}}\right. \\
& +\cos \theta-(\tan \alpha)(\sin \theta)]^{-1} .
\end{aligned}
$$

The exit radius of electron beam is also calculated by

$$
r_{\pi}=r_{0}\left[\frac{r_{0}\left(E-q \frac{V_{2} R_{2}-V_{1} R_{1}}{\Delta R}\right)+q\left(\frac{\Delta V}{\Delta R}\right) R_{1} R_{2}}{q\left(\frac{\Delta V}{\Delta R}\right) R_{1} R_{2} \tan ^{2} \alpha-r_{0}\left(E-q \frac{V_{2} R_{2}-V_{1} R_{1}}{\Delta R}\right)}\right] .
$$

For optimal focusing in HDA, the asymmetry and biasing parameters should be also taken into account. The asymmetry parameter of the analyzer, $\xi$ is described as $R_{\pi} / R$. The biasing parameter of the analyzer, $\gamma$ is calculated using $\gamma=1-q V\left(R_{0}\right) / E_{0}$. In an ideal centric HDA, $\xi$ and $\gamma$ parameters are usually taken to be 1 .

We use ANN method to analyze ideal field hemispherical deflector for the first time. ANN is a mathematical model having an advantage of new knowledge generation through its learning ability. ANN consists of input, hidden, and output layers. The input neurons from the input layer collect the training data while the output neurons generate the results. The neurons in different layers are connected to each other with many synaptic connections. The initial synaptic weights of the ANN are determined randomly. The ANN adjust the connection weights from examples of training dataset. By updating the synaptic weights, prediction performance of ANN is improved. Therefore, the unknown data can be predicted through the known examples [1-5].

\section{Results}

In this study, the trajectories of electron beam entering to hemispheres with launching angle $\left(\alpha=0^{\circ}\right)$ is predicted using the ANN. To train the network, the HDA geometry was chosen as $R_{1}=75 \mathrm{~mm}, R_{2}=125 \mathrm{~mm}$. In developed network, $E / E_{0}, R_{0}, R_{\pi}, \xi$, and $\gamma$ were determined as input parameters. The $\theta$ angles of electron trajectories within the hemispheres were also selected as input parameters and $r_{(\theta)}$ values selected as output parameters.
In this study, the back-propagation learning algorithm was used. Figure 2 represents the connection pattern of ANN.

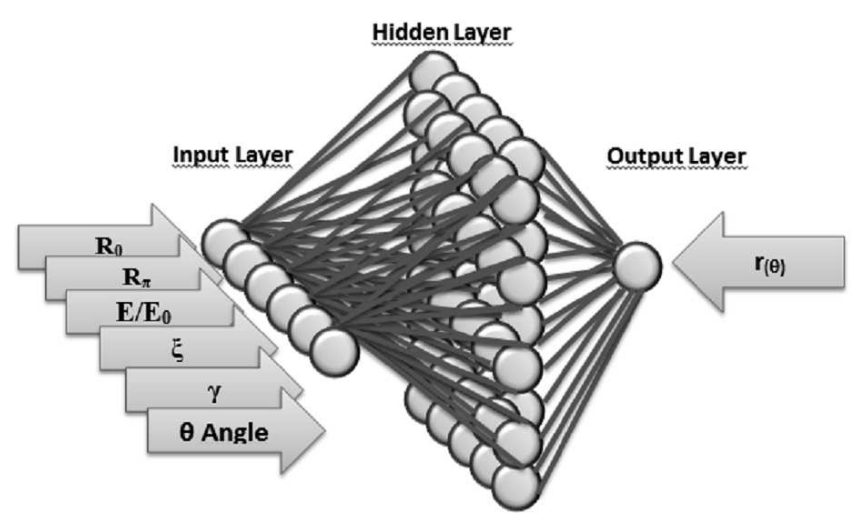

Fig. 2. The topology of developed network for the HDA having a geometry of $R_{1}=75 \mathrm{~mm}, R_{2}=125 \mathrm{~mm}$.

For training stage of ANN, optimum data sets were used. To generate optimum dataset, the entire training data obtained from SIMION 8.1 program was quality filtered.

TABLE I

Artificial neural network performance results.

\begin{tabular}{c|c|c|c|c}
\hline \hline \multirow{2}{*}{$\begin{array}{c}\text { ANN } \\
\text { architecture }\end{array}$} & \multicolumn{2}{|c|}{ Training dataset } & \multicolumn{2}{c}{ Testing dataset } \\
\cline { 2 - 5 } & MSE & R & MSE & R \\
\hline$(6$ 10, 1) & 0.0037 & 0.999 & 0.0038 & 0.999 \\
$(6$ 20, 1) & 0.0363 & 0.999 & 0.0386 & 0.999 \\
$(6$ 30, 1) & 0.2219 & 0.995 & 0.0465 & 0.999
\end{tabular}
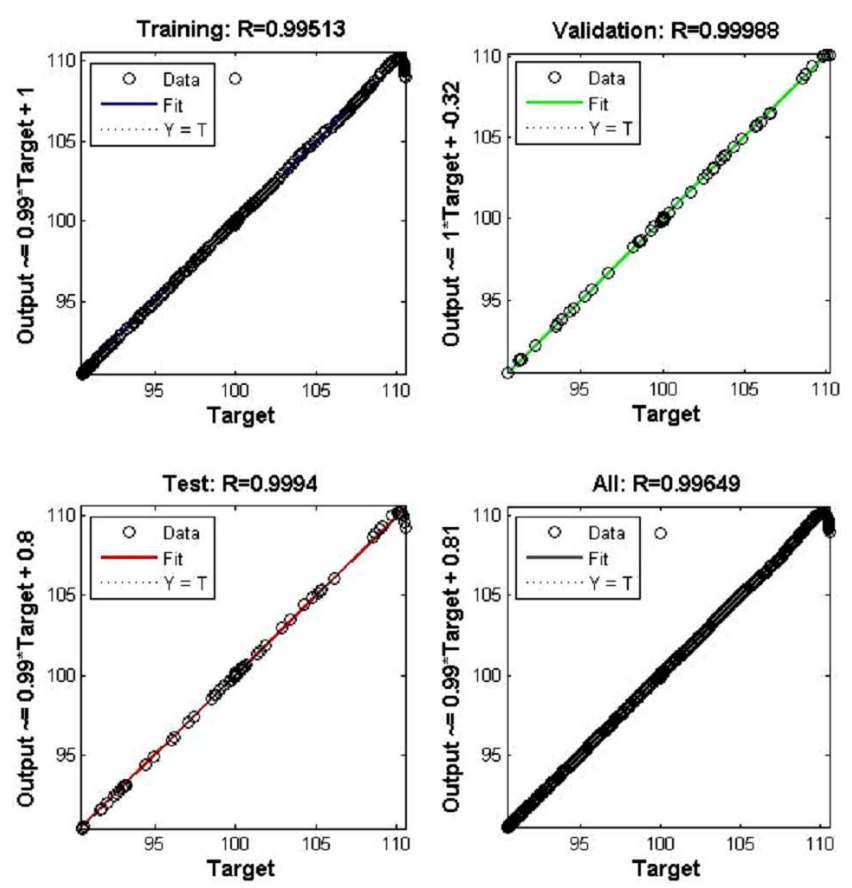

Fig. 3. Regression results of $(610,1)$ ANN architecture. 
For testing stage of ANN, test dataset consists of previously unknown data by ANN. Number of neurons in hidden layers was determined with the minimum error in results. The training stage is performed with the Levenberg-Marquardt algorithm. The hidden neuron activation function is hyperbolic tangent function $\left(\tanh x=\left(\mathrm{e}^{x}-\mathrm{e}^{-x}\right) /\left(\mathrm{e}^{x}+\mathrm{e}^{-x}\right)\right)$. The iteration number is limited to 20000. For three ANN architectures, analysis results are given in Table I and Fig. 3.

According to the MSE values of testing dataset, the $(610,1)$ ANN architecture has the best prediction performance. For a detailed analysis, the regression results of the $(610,1)$ ANN architecture are also given in Fig. 3. The regression results indicate that the ANN method is a perfect tool to predict the trajectories of electron beam within HDA.

\section{Conclusion}

ANN method is performed to investigate the electron trajectories in an HDA. The simulation dataset obtained from SIMION 8.1 simulation program is used as a training dataset for ANN architecture. High performance results indicate that $\mathrm{ANN}$ is useful and powerful method for hemispherical deflector analyzer. Different heuristic algorithm can be used for same dataset as a future study.

\section{Acknowledgments}

This research has been supported by Mehmet Akif Ersoy University Scientific Projects. Project numbers: NAP-0337-16 and NAP-0296-16.

\section{References}

[1] H. Wollnik, Nucl. Instrum. Methods 52, 250 (1967).

[2] D. Roy, J.D. Carette, Can. J. Phys. 49, 2118 (1971).

[3] R.E. Imhof, A. Adams, G.C. King, J. Phys. E Sci. Instrum. 9, 138 (1976).

[4] H. Ibach, Electron Spectroscopy for Surface Analysis, Springer-Verlag, Berlin 1977, p. 13.

[5] Y. Ballu, Applied Charged Particle Optics, Part B, Academic, New York 1980, p. 257.

[6] H. Goldstein, V. Twersky, Physics Today 5, 19 (1952).

[7] M.E. Rudd, in: Low Energy Electron Spectrometry, Ed. K.D. Sevier, Wiley, New York 1972, p. 17.

[8] E.M. Purcell, Phys. Rev. 54, 818 (1938).

[9] R. Herzog, Z. Phys. 97, 596 (1935).

[10] K. Jost, J. Phys. E Sci. Instrum. 12, 1001 (1979).

[11] B.A. Gurney, W. Ho, L.J. Richter, J.S. Villarrubia, Rev. Sci. Instrum. 59, 22 (1988).

[12] N. Mårtensson, P. Baltzer, P.A. Brühwiler, J.-O. Forsell, A. Nilsson, A. Stenborg, B. Wannberg, J. Electron Spectrosc. Relat. Phenom. 70, 117 (1994).

[13] O. Sise, G. Martinez, T.J.M. Zouros, M. Ulu, M. Dogan, J. Electron. Spectrosc. Relat. Phenom. 177, 42 (2010).

[14] A.H. Isik, Acta Phys. Pol. A 127, 1317 (2015).

[15] A.H. Isik, N. Isik, Acta Phys. Pol. A 129, 514 (2016).

[16] A.H. Isik, Acta Phys. Pol. A 127, 1717 (2015).

[17] N. Isik, Microsc. Microanal. 22, 458 (2016).

[18] N. Isik, A.H. Isik, Acta Phys. Pol. A 129, 628 (2016). 